PROCEEDINGS OF THE

AMERICAN MATHEMATICAL SOCIETY

Volume 126, Number 1, January 1998, Pages 53-60

S 0002-9939(98)04017-9

\title{
COMPLEXITY OF TENSOR PRODUCTS OF MODULES AND A THEOREM OF HUNEKE-WIEGAND
}

\author{
CLAUDIA MILLER
}

(Communicated by Wolmer V. Vasconcelos)

\begin{abstract}
This paper concerns the notion of complexity, a measure of the growth of the Betti numbers of a module. We show that over a complete intersection $R$ the complexity of the tensor product $M \otimes_{R} N$ of two finitely generated modules is the sum of the complexities of each if $\operatorname{Tor}_{i}^{R}(M, N)=0$ for $i \geq 1$. One of the applications is simplification of the proofs of central results in a paper of C. Huneke and R. Wiegand on the tensor product of modules and the rigidity of Tor.
\end{abstract}

\section{INTRODUCTION}

In this article we study the complexity of tensor products of modules over a complete intersection ring. Complexity is a measure of the growth of the Betti numbers of a module. We prove the following result; its main application is a simpler proof of some rigidity theorems of C. Huneke and R. Wiegand over a hypersurface.

(2.1) Proposition. If $M$ and $N$ are finitely generated modules over a complete intersection $R$ such that $\operatorname{Tor}_{i}^{R}(M, N)=0$ for $i>0$, then the complexity of the tensor product $M \otimes_{R} N$ is the sum of the complexities of $M$ and $N$.

A single argument proves two things: a special case of the proposition above, namely when $R$ is a hypersurface, and the theorem below.

(1.1) Theorem (Huneke-Wiegand, [HW2]). Let $R$ be a hypersurface, and let $M$ and $N$ be finitely generated $R$-modules. If $\operatorname{Tor}_{i}^{R}(M, N)=0$ for all $i>0$, then either $M$ or $N$ has finite projective dimension.

We found this result independently of Huneke and Wiegand, and we use it to simplify the proofs of central results (stated as (1.2), (1.3), and (1.4) in section 1 of this paper) in Huneke and Wiegand's first paper on tensor products of modules and the rigidity of Tor [HW1]. Their proof of (1.1) depends on (1.2), (1.3), and (1.4).

Remark. (1.1) can be thought of as a partial converse to the following theorem of Lichtenbaum.

Received by the editors March 25, 1996 and, in revised form, July 5, 1996

1991 Mathematics Subject Classification. Primary 13C14, 13C40, 13D05, 13D40, 13H10.

Key words and phrases. Complexity, complete intersection, hypersurface, rigidity, tensor product.

(C)1998 American Mathematical Society 
Theorem ([L], part of Theorem 3). Let $R$ be a ring which is the quotient of an unramified or equicharacteristic regular local ring by a principal ideal. Suppose $M$ is a finitely generated $R$-module of finite projective dimension. If $\operatorname{Tor}_{j}^{R}(M, N)=0$ for some $j>0$, then $\operatorname{Tor}_{i}^{R}(M, N)=0$ for all $i \geq j$.

In section 2 we turn to proving the general case of (2.1), and in section 3 we give the following application.

(3.1) Theorem. Let $R=S /(f)$ be a hypersurface of dimension $n$ with $n \geq 2$, and let $M$ be a finitely generated $R$-module. If the $(n-1)$-fold tensor product $M^{\otimes(n-1)}$ of $M$ is reflexive, then $M$ is free.

We present the material in the following order. Section 0 contains definitions, notation, and known results which we will use. Section 1 contains an elementary proof of the hypersurface result (1.1) of Huneke and Wiegand and the subsequent simplifications of the proofs of their other results (1.2), (1.3), and (1.4). In Section 2 we prove the general result for complete intersections, and in section 3 we apply it to prove (3.1).

\section{Preliminaries: Definitions, notation, AND Relevant Results}

We begin by reminding the reader of a few elementary definitions without specific reference. Most terms and concepts involved can be found in the text by Matsumura [M]. In this paper $R$ (or $S$ ) always denotes a local Noetherian commutative ring with maximal ideal $\mathfrak{m}=\mathfrak{m}_{R}$ (or $\mathfrak{m}_{S}$, respectively) and residue field $k$. Also, $M$ and $N$ denote finitely generated $R$-modules.

Definition. A complete intersection $R$ is a ring of the form $S /\left(f_{1}, \ldots, f_{t}\right)$, where $S$ is a regular local ring and $f_{1}, \ldots, f_{t}$ is an $S$-sequence in the maximal ideal of $S$. If $R$ can be written in this form with $t=1$, i.e., as $R=S /(f)$ for some non-zero $f \in \mathfrak{m}_{S}$, it is called a hypersurface.

Definition. The embedding dimension, denoted by $\operatorname{embdim} R$, of a local ring $(R, \mathfrak{m}, k)$ is the dimension of the $k$-vector space $\mathfrak{m} / \mathfrak{m}^{2}$. The codimension of $R$, denoted by $\operatorname{codim} R$, is the nonnegative integer embdim $R-\operatorname{dim} R$. If $R$ is a complete intersection, the codimension of $R$ is also given by the formula

$\operatorname{codim} R=\inf \left\{t \mid R \cong S /\left(f_{1}, \cdots, f_{t}\right), S\right.$ a regular local ring,

$$
\left.f_{1}, \cdots, f_{t} \text { an S-sequence }\right\} \text {. }
$$

Definition. A ring is said to satisfy $R_{i}$ if, for each prime ideal $P$ of height less than or equal to $i$, the ring $R_{P}$ is regular. A finitely generated $R$-module $M$ satisfies $S_{i}$ if $\operatorname{depth}_{R_{P}}\left(M_{P}\right) \geq \min (i$, ht $P)$ for each prime ideal $P$ of $R$. A ring satisfies $S_{i}$ if it does so as a module over itself.

Definition. Let $M$ be a finitely generated $R$-module. For $n \geq 0$, the $n$-th Betti number, denoted by $b_{n}(M)$, is the rank of the free module $F_{i}$ in a minimal free resolution $F_{\bullet}$ of $M$. Equivalently,

$$
b_{n}(M)=\operatorname{dim}_{k}\left(\operatorname{Tor}_{n}^{R}(M, k)\right) .
$$

We remark that $b_{n}\left(\operatorname{syz}_{i}(M)\right)=b_{n+i}(M)$ for all $n \geq 0$ and $i \geq 0$, where $\operatorname{syz}_{i}(M)$ denotes the $i^{t h}$ syzygy in a minimal free resolution of $M$. 
Definition. The Poincaré series of $M$, denoted by $P_{M}(t)$, is the generating function for the sequence of Betti numbers, that is,

$$
P_{M}(t)=\sum_{n=0}^{\infty} b_{n}(M) t^{n} \in \mathbb{Z}[[t]]
$$

A measure of the growth of Betti numbers is given by complexity:

Definition. The complexity, $\mathrm{cx}_{R} M$, of an $R$-module $M$ is the least integer $c \geq 0$ such that, for $n \gg 0, b_{n}(M) \leq \gamma n^{c-1}$ for some real number $\gamma$.

An $R$-module $M$ satisfies $\operatorname{cx}_{R} M=0$ exactly when it has finite projective dimension; $M$ satisfies $\mathrm{cx}_{R} M \leq 1$ exactly when it has bounded Betti numbers. The complexity of a module may be infinite. However, the following theorem of Eisenbud implies that the complexity of a module over a hypersurface is at most one.

(0.1) Theorem ([E], (6.1)). Let $R$ be a hypersurface. Then the minimal free resolution of any finitely generated $R$-module becomes periodic of period two after $\operatorname{dim} R+1$ steps.

More generally, the following result tells us that the complexity of a module over a complete intersection is finite (see $[\mathrm{Gu}],(4.2)$ or $[\mathrm{Av}],(3.1)$, Remark 3 ).

(0.2) Proposition ([Gu]). A finitely generated module over a complete intersection of codimension thas complexity at most $t$.

In fact, more is known to be true about the complexity of a module; to give the statement in its full generality, we need a few preliminary definitions.

Notation. Let $R$ be a local Noetherian ring. If $k=R / \mathfrak{m}$ is infinite, let $\widetilde{R}$ be the $\mathfrak{m}$-adic completion $\widehat{R}$ of $R$; if $k$ is finite, let $\widetilde{R}$ be the completion $\left(R\left[\widehat{X]_{\mathfrak{m} R}[X]}\right)\right.$. Let $\widetilde{M}$ be the $\widetilde{R}$-module $M \otimes_{R} \widetilde{R}$.

Since $\widetilde{R}$ is a faithfully flat extension of $R$, the quantities depth, dimension, Betti numbers, and complexity remain the same under the base change from $R$ to $\widetilde{R}$.

Definition. A deformation of $R$ is a local ring $Q$ with a $Q$-sequence $\underline{\mathbf{x}}$ such that $R \cong Q /(\underline{\mathbf{x}})$.

Definition. The virtual projective dimension of $M$, denoted by $\operatorname{vpd}_{R}(M)$, is defined to be

$$
\operatorname{vpd}_{R}(M)=\min \left\{\operatorname{pd}_{Q} \widetilde{M} \mid Q \text { is a deformation of } \widetilde{R}\right\} .
$$

Remarks. Any finitely generated module over a complete intersection has finite virtual projective dimension ([Av], (3.4.4)). Furthermore, any module which has finite virtual projective dimension has finite complexity ([Av], (3.1), Remark 3).

Now we are in a position to state the following result of Avramov which provides us with an equivalent definition of complexity for modules of finite virtual projective dimension, in particular, for any finitely generated module over a complete intersection.

(0.3) Theorem $([\mathrm{Av}],(3.12))$. Let $M$ be a module of finite virtual projective dimension. Then the complexity $\operatorname{cx}(M)$ equals the order of the pole of the Poincaré series $P_{M}(t)$ at $t=1$. 
We will use two more results, the first being the following one of Auslander.

(0.4) Theorem ([Au], (1.2)). Let $M$ and $N$ be non-zero modules over the local ring $R$ such that $\operatorname{pd}(M)<\infty$. Let $q$ be the largest integer such that $\operatorname{Tor}_{q}^{R}(M, N) \neq$ 0 . If either $\operatorname{depth}\left(\operatorname{Tor}_{q}^{R}(M, N)\right) \leq 1$ or $q=0$, then we have

$$
\operatorname{depth}(N)=\operatorname{depth}\left(\operatorname{Tor}_{q}^{R}(M, N)\right)+\operatorname{pd}(M)-q .
$$

The other result is the following one of Jorgensen.

(0.5) Theorem $([\mathrm{J}],(1.3))$. Let $R$ be a complete intersection of codimension $r>0$ with infinite residue field. Suppose $\mathfrak{F}$ is a finite set of $R$-modules. Then there exist a complete intersection $R_{1}$ of codimension $r-1$ and an element $x \in R_{1}$ such that $R_{1} / x R_{1} \cong R$ and such that, for all $M \in \mathfrak{F}$,

$$
\operatorname{cx}_{R_{1}}(M)=\left\{\begin{array}{cl}
\operatorname{cx}_{R}(M)-1 & , \text { if } \operatorname{cx}_{R}(M)>0 \\
0 & , \text { if } \operatorname{cx}_{R}(M)=0 .
\end{array}\right\}
$$

\section{Result over HyPERSURFACES AND APPLICATION TO A THEOREM OF HUNEKE AND WiEgAND}

What can be said about two modules $M$ and $N$ such that $\operatorname{Tor}_{i}^{R}(M, N)$ vanishes for all positive $i$ ? We explore the question when $R$ is a hypersurface ring first. We give an elementary proof of the following theorem, which was found independently by us at the same time that Huneke and Wiegand found it.

(1.1) Theorem ([HW2]). Let $R$ be a hypersurface. If $\operatorname{Tor}_{i}^{R}(M, N)=0$ for all $i \gg 0$, then one of $M$ or $N$ has finite projective dimension.

Proof. By taking the $j^{\text {th }}$ syzygy of $M$ for a sufficiently large integer $j$, we may assume that $\operatorname{Tor}_{i}^{R}(M, N)=0$ for all $i>0$. Let $P_{\bullet} \longrightarrow M$ and $Q_{\bullet} \longrightarrow N$ be minimal free resolutions of $M$ and $N$, respectively, over $R$. Consider $P_{\bullet} \otimes Q_{\bullet}$. Since $\operatorname{Tor}_{i}(M, N)=0$ for all $i>0$,

$$
\mathrm{H}_{i}\left(P_{\bullet} \otimes Q_{\bullet}\right)=\left\{\begin{array}{cc}
M \otimes N & , n=0 \\
0 & , n>0 .
\end{array}\right\}
$$

Also, all the differential maps in $P_{\bullet} \otimes Q_{\bullet}$ are minimal. So, $P_{\bullet} \otimes Q_{\bullet}$ is a minimal free resolution of $M \otimes N$.

If $P_{\bullet}$ and $Q_{\bullet}$ are infinite complexes, then we have the following inequality:

$$
b_{n}(M \otimes N)=\sum_{i+j=n} \operatorname{rank}\left(P_{i} \otimes Q_{n-i}\right) \geq n+1 .
$$

This is a contradiction since by (0.1) the Betti numbers of $M \otimes N$ are bounded. So, indeed, either $M$ or $N$ has finite projective dimension.

Huneke and Wiegand do not state the above result explicitly until their second paper on the topic [HW2], in which they deduce it from the following three results in their first paper [HW1].

(1.2) Proposition ([HW1], (2.5)). Let $R$ be a complete intersection. If

$$
\operatorname{Tor}_{i}^{R}(M, N)=0 \quad \text { for all } i>0,
$$

then

$$
\operatorname{depth}(M)+\operatorname{depth}(N)=\operatorname{depth}(R)+\operatorname{depth}(M \otimes N) .
$$


(1.3) Second Rigidity Theorem ([HW1], (2.7)). Let $R=S /(f)$ be a hypersurface. If $M \otimes N$ is reflexive, then $M$ and $N$ are reflexive and $\operatorname{Tor}_{i}^{R}(M, N)=0$ for all $i>0$.

(1.4) Theorem ([HW1], (3.1)). Let $R=S /(f)$ be a hypersurface. If $M \otimes N$ is maximal Cohen-Macaulay, then at least one of $M$ or $N$ is free (and the other maximal Cohen-Macaulay).

They first prove (1.2). Then, in order to prove the Second Rigidity Theorem (1.3), they need the one-dimensional case of (1.4). They obtain it from a first rigidity theorem of the paper and some special dimension one ring theory. Then they use the Second Rigidity Theorem to implement the inductive step in proving the higher dimensional result of (1.4).

Since our proof of Theorem (1.1) is independent of (1.2), (1.3), and (1.4), we know a priori that one of the modules has finite projective dimension whenever all the Tor's vanish. This additional piece of information enables us to simplify the proof of those three results, as we shall see now.

Proof of (1.2) for a hypersurface. By (1.1), one of the modules, say $M$, has finite projective dimension. Then by (0.4) (with $q=0$ ), we have

$$
\operatorname{depth}(N)=\operatorname{depth}(M \otimes N)+\operatorname{pd}(M) .
$$

By the Auslander-Buchsbaum formula, $\operatorname{pd}(M)=\operatorname{depth}(R)-\operatorname{depth}(M)$, and so we are done.

Theorem (1.1) also simplifies the proof of the one-dimensional case of (1.4) (stated in [HW1] as (3.7)) as follows:

Proof of the One-Dimensional Case of (1.4). As they showed, one may assume $R$ is a hypersurface. They prove, using their first rigidity result, that $\operatorname{Tor}_{i}^{R}(M, N)=0$ for all $i>0$. By (1.2), we have the relation

$$
\operatorname{depth}(M)+\operatorname{depth}(N)=1+\operatorname{depth}(M \otimes N) .
$$

Since the right-hand side equals two by hypothesis, one must have $\operatorname{depth}(M)=$ $\operatorname{depth}(N)=1$. Also (1.1) gives that one of $M$ or $N$ has finite projective dimension and so is free by the Auslander-Buchsbaum formula.

This releases the Second Rigidity Theorem (1.3) from its dependence on the dimension one ring theory of $\S 3$ of [HW1] originally needed for the proof of this one-dimensional case.

Using (1.1) again and the Second Rigidity Theorem, we get (1.4) as an immediate corollary as follows.

Proof of (1.4). Since $M \otimes N$ is reflexive, the Second Rigidity Theorem implies that $\operatorname{Tor}_{i}^{R}(M, N)=0$ for all $i>0$. So, by (1.2), both $M$ and $N$ have depth equal to the depth of $R$. By (1.1), one of $M$ or $N$ has finite projective dimension and so is free by the Auslander-Buchsbaum formula. 


\section{Generalization to complete intersections}

Suppose two modules $M$ and $N$ have the property that $\operatorname{Tor}_{i}^{R}(M, N)=0$ for all $i \gg 0$. If $R$ is a hypersurface, we found that this condition forces one of $M$ or $N$ to have finite projective dimension, that is, zero complexity (Theorem (1.1)). What can be concluded if $R$ is a complete intersection? The key to a generalization of Theorem (1.1) to cover these rings is the following result.

(2.1) Proposition. If $M$ and $N$ are modules over a complete intersection $R$ such that $\operatorname{Tor}_{i}^{R}(M, N)=0$ for all $i>0$, then $\operatorname{cx}_{R}(M \otimes N)=\operatorname{cx}_{R}(M)+\operatorname{cx}_{R}(N)$.

Proof. Let $P_{\bullet} \longrightarrow M$ and $Q_{\bullet} \longrightarrow N$ be minimal free resolutions of $M$ and $N$, respectively, over $R$. Consider $P \bullet \otimes Q$. Since $\operatorname{Tor}_{i}(M, N)=0$ for all $i>0$,

$$
\mathrm{H}_{i}\left(P_{\bullet} \otimes Q_{\bullet}\right)=\left\{\begin{array}{cc}
M \otimes N & , n=0 \\
0 & , n>0 .
\end{array}\right\}
$$

Also, all the differential maps in $P_{\bullet} \otimes Q_{\bullet}$ are minimal. So, $P_{\bullet} \otimes Q_{\bullet}$ is a minimal free resolution of $M \otimes N$. Therefore,

$$
\begin{aligned}
b_{n}(M \otimes N) & =\operatorname{rank}\left(P_{\bullet} \otimes Q_{\bullet}\right)_{n} \\
& =\sum_{i+j=n} b_{i}(M) b_{j}(N) \\
& =\sum_{i=0}^{n} b_{i}(M) b_{n-i}(N) .
\end{aligned}
$$

This formula gives the relation

$$
P_{M \otimes N}(t)=P_{M}(t) \cdot P_{N}(t)
$$

between the Poincaré series. Then by counting the order of the pole at $t=1$ of each series and applying (0.3), we get $\operatorname{cx}_{R}(M \otimes N)=\operatorname{cx}_{R}(M)+\operatorname{cx}_{R}(N)$.

The same proof for any two modules of finite virtual projective dimension gives the following result.

(2.2) Proposition. Let $M$ and $N$ be modules of finite virtual projective dimension over a local ring $R$ such that $\operatorname{Tor}_{i}^{R}(M, N)=0$ for all $i>0$. Then $\operatorname{cx}_{R}(M \otimes N)=$ $\mathrm{cx}_{R}(M)+\mathrm{cx}_{R}(N)$.

Remark. For a hypersurface, Proposition (2.1) has Theorem (1.1) as an immediate corollary: the complexity of $M \otimes N$ is at most one by $(0.2)$, but then $\operatorname{cx}_{R}(M \otimes N)=$ $\mathrm{cx}_{R}(M)+\mathrm{cx}_{R}(N)$ implies that one of $M$ or $N$ has zero complexity, i.e., finite projective dimension.

For a complete intersection of codimension larger than one, (2.1) has the following corollaries:

(2.3) Corollary. Let $R=S /\left(f_{1}, f_{2}\right)$ be a complete intersection of codimension two. If $\operatorname{Tor}_{i}^{R}(M, N)=0$ for all $i>0$, then one of the following statements holds.

a) $M$ and $N$ have periodic resolutions of period two over $R$.

b) Either $M$ or $N$ has finite projective dimension over $R$.

Proof. By (2.1), we have $\mathrm{cx}_{R}(M \otimes N)=\mathrm{cx}_{R}(M)+\mathrm{cx}_{R}(N)$, and by $(0.2)$ we have $\mathrm{cx}_{R}(M \otimes N) \leq 2$. If both modules have complexity one, then they have periodic resolutions by (0.1). If not, then either $M$ or $N$ has complexity zero, that is, finite projective dimension. 
(2.4) Corollary. Let $R$ be a complete intersection of codimension $t \geq 2$ with infinite residue field. If $\operatorname{Tor}_{i}^{R}(M, N)=0$ for all $i>0$, then one of the following statements holds.

a) $R$ can be written as $S /\left(f_{1}, f_{2}, \ldots, f_{t}\right)$ where $S$ is a regular local ring and $f_{1}, f_{2}, \ldots, f_{t}$ an $S$-sequence such that both $M$ and $N$ have finite projective dimension over $S /\left(f_{1}\right)$.

b) Either $M$ or $N$ has finite projective dimension over $R$.

Proof. By (2.1) and (0.2), we see that

$$
\mathrm{cx}_{R}(M)+\mathrm{cx}_{R}(N)=\mathrm{cx}_{R}(M \otimes N) \leq t .
$$

So, either both modules have complexity at most $t-1$, or one of the modules has complexity zero, i.e., finite projective dimension. In the first case, the desired result follows by applying (0.5).

Remark 1. Each of the corollaries above holds with the modified hypothesis that there exists an integer $i_{0}$ such that $\operatorname{Tor}_{i}^{R}(M, N)=0$ for all $i>i_{0}$. One just applies the corollaries to $M$ and $\operatorname{syz}_{i_{0}}^{R}(N)$, noting the equalities $\operatorname{Tor}_{i}\left(M, \operatorname{syz}_{i_{0}}^{R}(N)\right)=$ $\operatorname{Tor}_{i+i_{0}}(M, N)$ and $\mathrm{cx}_{R}(N)=\operatorname{cx}_{R}\left(\operatorname{syz}_{i_{0}}^{R}(N)\right)$.

\section{Another application}

Another application of (2.1) is the following result.

(3.1) Theorem. Let $R=S /(f)$ be a hypersurface of dimension $n$ with $n \geq 3$. If the $(n-1)$-fold tensor product $M^{\otimes(n-1)}$ of $M$ is reflexive, then $M$ is free.

Proof. By repeated application of the Second Rigidity Theorem (1.3), we conclude that $M$ has finite projective dimension. Let $P_{\bullet} \longrightarrow M$ be a minimal free resolution of $M$. Since $\operatorname{Tor}_{i}\left(M, M^{\otimes r}\right)=0$ for all $r>0$ such that $1+r \leq n-1$ and all $i>0$, we get that

$$
P_{\bullet}^{\otimes(n-1)} \longrightarrow M^{\otimes(n-1)}
$$

is a minimal free resolution. Its length is $(n-1) \operatorname{pd}(M)$. So,

$$
(n-1) \operatorname{pd}(M)=\operatorname{pd}\left(M^{\otimes(n-1)}\right)<\infty
$$

and by the Auslander-Buchsbaum formula, this is equal to $n-\operatorname{depth}\left(M^{\otimes(n-1)}\right)$, which in turn is less than $n-2$ since $M^{\otimes(n-1)}$ is reflexive and $n \geq 2$. Then, since $\operatorname{pd}(M)$ is a nonnegative integer and $n \geq 2$, we must have $\operatorname{pd}(M)=0$, that is, $M$ is free.

More generally, we have the following proposition:

(3.2) Proposition. Let $R$ be a complete intersection of codimension $t$ and dimension $n$.

a) If $\operatorname{Tor}_{i}\left(M, M^{\otimes k}\right)=0$ for all $0<k \leq t-1$ and all $i>0$, then $M$ has a periodic resolution.

b) If $\operatorname{Tor}_{i}\left(M, M^{\otimes k}\right)=0$ for all $0<k \leq t$ and all $i>0$, then $\operatorname{pd}_{R}(M)<\infty$.

c) If $\operatorname{Tor}_{i}\left(M, M^{\otimes k}\right)=0$ for all $0<k \leq \max (t, n)$ and all $i>0$, then $M$ is free.

Proof. Repeated application of (2.1) gives, in case a),

$$
t \operatorname{cx}_{R}(M)=\operatorname{cx}\left(M^{\otimes t}\right) \leq t, \quad \text { i.e. } \quad \operatorname{cx}_{R}(M) \leq 1,
$$


and in cases b) and c),

$$
(t+1) \operatorname{cx}_{R}(M)=\operatorname{cx}\left(M^{\otimes(t+1)}\right) \leq t, \quad \text { i.e. } \quad \operatorname{cx}_{R}(M)=0 .
$$

Also, for case c), since $\operatorname{pd}_{R}(M)<\infty, \operatorname{Tor}_{i}\left(M, M^{\otimes k}\right)=0$ for $0<k \leq n$ and all $i>0$ implies that

$$
(n+1) \operatorname{pd}(M)=\operatorname{pd}\left(M^{\otimes(t+1)}\right) \leq n
$$

as in the previous proposition, and so $\operatorname{pd}_{R}(M)=0$, i.e., $M$ is free.

\section{REFERENCES}

[Au] M. Auslander, Modules over unramified regular local rings, Ill. J. Math. 5 (1961), 631-647. MR 31:3460

[Av] L. Avramov, Modules of finite virtual projective dimension, Invent. Math. 96 (1989), 71101. MR 90g: 13027

[E] D. Eisenbud, Homological algebra on a complete intersection, with an application to group representations, Trans. Amer. Math. Soc. 260 (1980), 35-64. MR 82d:13013

[Gu] T. Gulliksen, A change of rings theorem, with applications to Poincaré series and intersection multiplicity, Math. Scand. 34 (1974), 167-183. MR 51:487

[HW1] C. Huneke and R. Wiegand, Tensor products of modules and the rigidity of Tor, Math. Ann. 299 (1994), 449-476. MR 95m:13008

[HW2] C. Huneke and R. Wiegand, Tensor products of modules, rigidity, and local cohomology, submitted.

[J] D. Jorgensen, Complexity and Tor on a complete intersection, submitted.

[L] S. Lichtenbaum, On the vanishing of Tor in regular local rings, Ill. J. Math. 10 (1966), 220-226. MR 32:5688

[M] H. Matsumura, Commutative Ring Theory, in Cambridge Studies in Advanced Mathematics, no. 8, Cambridge Univ. Press, Cambridge, 1989. MR 90i:13001

Department of Mathematics, University of Illinois, Urbana, Illinois 61801

E-mail address: cmiller@math.uiuc.edu

Current address: Department of Mathematics, University of Michigan, Ann Arbor, Michigan 48109 